\title{
Do the terms innate and adaptive immunity create conceptual barriers?
}

\author{
Lewis Lanier and Joseph Sun suggest that new data showing that innate immune cells can \\ have memory characteristics make a strict binary classification between innate and adaptive \\ immunity meaningless.
}

What is the difference between innate and adaptive immunity? Traditionally, innate immunity was assumed to be rapid, non-specific and identical qualitatively and quantitatively each time the same pathogen was encountered. Many innate immune cells are thought to be short-lived - for example, the lifespan of a neutrophil is estimated to be a few hours or days - making memory an irrelevant concept. Conversely, the hallmarks of adaptive immunity are thought to include the generation of long-lived, antigen-specific cells after initial exposure to an antigen or pathogen that can respond faster and more robustly on subsequent encounters with the same antigen or pathogen. Adaptive immunity is generally considered to be the exclusive domain of B and T cells; granulocytes, monocytes, macrophages, dendritic cells and natural killer (NK) cells have been delegated to the innate immune system, which also comprises epithelial cell barriers, complement, antimicrobial peptides and other soluble factors.

Recently, however, the distinctions between innate and adaptive immunity have become blurred. Certain subsets of B and T cells, such as B1 cells, $\gamma \delta$ T cells and invariant NKT (iNKT) cells, are often referred to as innate-like lymphocytes ${ }^{1}$. The phenotype of such cells in naive animals is often more similar to that of effector cells than that of small, resting lymphocytes that have not previously encountered an antigen or pathogen. This is reflected by their constitutive expression of certain activation or memory cell surface markers and by their ability to mediate effector functions such as cytolytic activity or cytokine production rapidly after stimulation through their antigen receptors. The antigen receptor repertoire of these innate-like lymphocytes, including iNKT cells and some subsets of $\gamma \delta$ T cells, might also be more restricted than that of conventional T cells.

Reciprocally, certain innate immune cells also seem not to fit the conventions. Our recent studies have shown that NK cells, which are considered to be lymphocytes of the innate immune system, have several features that are normally attributed exclusively to cells of the adaptive immune system ${ }^{2,3}$. Mouse NK cells that express the germline-encoded invariant receptor $\mathrm{LY} 49 \mathrm{H}$, which specifically recognizes the $\mathrm{m} 157$ glycoprotein encoded by cytomegalovirus $(\mathrm{CMV})^{4}$, clonally expand during viral infection, and the population contracts after the infection is controlled ${ }^{5}$. Surprisingly, a subset of these CMV-specific NK cells can persist in the host for months after infection and can show a 'recall' response, resulting in the marked clonal expansion of cells that are specific for m157 after a second exposure to $\mathrm{CMV}^{3}$. Although these 'memory' NK cells proliferate with similar kinetics to naive NK cells after infection, they have a more robust cytolytic and cytokine response and protect the host more efficiently than naive NK cells. Therefore, the fundamental behaviour of conventional $\mathrm{CD}^{+} \mathrm{T}$ cells and NK cells during $\mathrm{CMV}$ infection is remarkably similar. One important difference however is that before immunization, $\mathrm{CD}^{+} \mathrm{T}$ cells that are specific for any particular antigenic determinant are present at exceedingly low precursor frequencies in naive hosts (typically estimated at $\leq 1$ in 100,000 T cells), whereas the CMV-specific $\mathrm{LY} 49 \mathrm{H}^{+} \mathrm{NK}$ cells are present at high precursor frequencies (comprising about $50 \%$ of NK cells in C57BL/6 mice) before infection.

So, what are the distinguishing features of memory cells? First, memory implies that an antigen-activated cell or its clonal progeny can survive for a long time after the pathogen or antigen has been cleared from the host. These cells must then be able to respond to re-exposure to the same antigen or pathogen in a qualitatively different manner than naive cells. The recall response can be reflected by faster proliferation or more robust effector functions, such as secreting higher levels of cytokines and chemokines or producing them more quickly, or in the case of cytotoxic cells, as being able to kill target cells sooner or more efficiently after a second encounter with the specific antigen or pathogen. Although it is generally assumed that memory $\mathrm{T}$ cells proliferate faster than naive $\mathrm{T}$ cells after antigen-specific stimulation, studies using $\mathrm{T}$ cell receptor (TCR)-transgenic mouse models have shown that, at least for certain types of $\mathrm{T}$ cell, the kinetics of proliferation are essentially identical for naive and memory $\mathrm{T}$ cells 6 . The difference in the response is largely accounted for by a higher frequency of memory than naive $\mathrm{T}$ cells that are specific for a particular antigen. With respect to effector functions, memory $\mathrm{T}$ cells are more efficient at mediating cytokine 
production and cytotoxicity after TCR ligation than naive T cells. Furthermore, previous studies have documented qualitative differences between naive, effector and memory T cells using transcriptional profiling techniques? ${ }^{7}$.

When considering the role of memory cells in an immune response, one typically thinks of their actions after they are re-challenged with the same pathogen or antigen. The vigorous clonal expansion of memory B or $\mathrm{T}$ cells is indeed antigen specific; however, an underappreciated aspect of memory cells is that they can also be activated and contribute to immune responses in a nonantigen-specific manner. For example, memory B cells can proliferate and secrete antibody when stimulated by $\mathrm{CpG}$ containing DNA (through Toll-like receptor 9; TLR9) and interleukin-15 (IL-15) without requiring cognate antigen, whereas naive B cells are unresponsive to CpG DNA and IL-15 in the absence of antigen ${ }^{8}$. Similarly, unlike naive T cells, memory T cells can secrete interferon- $\gamma($ IFN $\gamma)$ in a non-antigen-specific manner. During bacterial infection, memory T cells, but not naive T cells, rapidly secrete IFN $\gamma$ in response to IL-12 and IL-18 produced by dendritic cells in the absence of cognate antigen ${ }^{9,10}$. Moreover, when mice are injected with polyinosinic:polycytidylic acid (poly I:C; which induces the production of type I IFNs by plasmacytoid dendritic cells following their stimulation through TLR9) or endotoxin (which stimulates myeloid cells through TLR4 to produce IL-12 and IL-18), memory $\mathrm{T}$ cells, but not naive T cells, secrete IFN $\gamma$ in the absence of cognate antigen ${ }^{11,12}$.

Similar to memory $\mathrm{T}$ cells, the ability of memory $\mathrm{LY} 49 \mathrm{H}^{+} \mathrm{NK}$ cells to undergo clonal expansion is strictly antigen specific in that re-challenge with CMV lacking m157 fails to drive proliferation. However, like memory $\mathrm{T}$ cells and memory B cells, when memory NK cells are stimulated through other non-antigen-specific receptors, they produce more cytokines than naive NK cells. Therefore, the 'wiring' of memory cells in the B, T and NK cell lineages is different to that of naive cells, allowing the memory cells to participate in an immune response not only against the previously encountered cognate antigen, but also against other infections in a bystander manner. This ability of memory cells to rapidly and nonspecifically participate during subsequent infection provides chemokines and cytokines such as IFN $\gamma$ to activate local dendritic cells, macrophages and granulocytes and might help to recruit and augment the differentiation of naive antigen-specific cells.

The recent studies suggesting the existence of memory NK cells should prompt a re-evaluation of other innate immune cells. Certain populations of myeloid cells, for example Langerhans cells, have been shown to be longlived in mice $^{13}$. After myeloid cells are initially stimulated through their TLRs, how long does this activation state last? Do these myeloid cells adapt, acquiring a long-lived alteration in their kinetics of response or transcriptional signature when they are stimulated at a later time either through the same receptors or receptors not involved in the previous immune response? Phagocytic cells in Drosophila melanogaster apparently can 'remember' previous infections, having specificity for only some bacteria $^{14}$. There is evidence for an enhanced immune response against microbial pathogens following a second encounter in several invertebrates, including water fleas, copepods, red flour beetles, bumblebees and cockroaches, and an increased second-set rejection of allogeneic colonies has been shown in tunicates, sea urchins and sponges ${ }^{15}$. Therefore, the ability of the immune system to behave differently on a second encounter with the same pathogen or allogeneic organism seems to pre-date the evolution of cells with the ability to generate antigenspecific receptors by somatic gene recombination.

In the end, what distinguishes innate and adaptive immunity? Is a qualitatively distinct response of an immune cell after a second stimulation sufficient to consider it adaptive? How long after the clearance of a pathogen or antigen do the responding immune cells need to persist and display a qualitatively distinct response to re-challenge to be considered memory cells? If the definition of adaptive immunity is that the cells require productive somatic gene rearrangement of their primary receptor for antigen, whereas innate immune cells solely rely on invariant, germline-encoded receptors for sensing pathogens, then the distinction between innate and adaptive immunity is unambiguous. However, when antigen-specific B cells and memory $\mathrm{T}$ cells mediate non-antigen-specific responses, often using innate-like receptors, and when innate immune cells display many of the hallmarks of adaptive immune cells, a strict binary classification becomes rather meaningless. Instead of arguing on semantics, it will be more important to understand these processes and learn to harness them for more efficient control of infectious disease, possibly providing new strategies for vaccination.

\footnotetext{
Bendelac, A. Innate-like lymphocytes. Curr. Opin. Immunol. 18 517-518 (2006)

2. O'Leary, J. G., Goodarzi, M., Drayton, D. L. \& von Andrian, U. H. $\mathrm{T}$ cell- and $\mathrm{B}$ cell-independent adaptive immunity mediated by natural killer cells. Nature Immunol. 7, 507-516 (2006).

3. Sun, J. C., Beilke, J. N. \& Lanier, L. L. Adaptive immune features of natural killer cells. Nature 457, 557-561 (2009).

4. Arase, H., Mocarski, E. S., Campbell, A. E., Hill, A. B. \& Lanier, L. L. Direct recognition of cytomegalovirus by activating and inhibitory NK cell receptors. Science 296, 1323-1326 (2002).

5. Dokun, A. O. et al. Specific and nonspecific NK cell activation during virus infection. Nature Immunol. 2, 951-956 (2001).

6. Zimmermann, C., Prevost-Blondel, A., Blaser, C. \& Pircher, H. Kinetics of the response of naive and memory CD8 T cells to antigen: similarities and differences. Eur. J. Immunol. 29, 284-290 (1999).

7. Kaech, S. M., Hemby, S., Kersh, E. \& Ahmed, R. Molecular and functional profiling of memory CD8 T cell differentiation. Cell 111 , 837-851 (2002)

8. Bernasconi, N. L., Traggiai, E. \& Lanzavecchia, A. Maintenance of serological memory by polyclonal activation of human memory B cells. Science 298, 2199-2202 (2002)

9. Lertmemongkolchai, G., Cai, G., Hunter, C. A. \& Bancroft, G. J. Bystander activation of $\mathrm{CD} 8{ }^{+} \mathrm{T}$ cells contributes to the rapid production of IFN- $\gamma$ in response to bacterial pathogens. J. Immunol. 166, 1097-1105 (2001).

10. Berg, R. E., Crossley, E., Murray, S. \& Forman, J. Memory CD8+ $\mathrm{T}$ cells provide innate immune protection against Listeria monocytogenes in the absence of cognate antigen. J. Exp. Med. 198, 1583-1593 (2003).

11. Tough, D. F., Borrow, P. \& Sprent, J. Induction of bystander T cell proliferation by viruses and type I interferon in vivo. Science 272 1947-1950 (1996).

12. Kambayashi, T., Assarsson, E., Lukacher, A. E., Ljunggren, H. G. \& Jensen, P. E. Memory CD8 ${ }^{+}$T cells provide an early source of IFN- $\gamma$. J. Immunol. 170, 2399-2408 (2003).

13. Merad, M. et al. Langerhans cells renew in the skin throughout life under steady-state conditions. Nature Immunol. 3, 1135-1141 (2002).

14. Pham, L. N., Dionne, M. S., Shirasu-Hiza, M. \& Schneider, D. S. A specific primed immune response in Drosophila is dependent on phagocytes. PLoS Pathog. 3 e26 (2007).

15. Kurtz, J. Specific memory within innate immune systems. Trends Immunol. 26, 186-192 (2005).
} 